\title{
The in vacuo release of Ar from hydrous and anhydrous minerals
}

\section{IGOR MARIA VILLA}

Università di Milano Bicocca, Universität Bern

Presenting Author: igor.villa@unimib.it

Understanding the systematics of Ar release during in vacuo stepwise heating is crucial for reliable geochronological interpretations. Hydrous mineral geochronometers (amphiboles, micas) are known to crystallographers to modify their structure at the atomic scale when heated above their dehydration threshold. Their in vacuo degassing does not follow Fick's Law in an inert, homogeneous matrix, and instead proceeds from the structural collapse during dehydration. Moreover, they are practically never monomineralic: EPMA element maps document ubiquitous intergrowths with retrogression/alteration products at a scale $<20 \mu \mathrm{m}$ that escape recognition during handpicking. Polymineralic mixtures in practically all step-heated mineral separates are independently confirmed by common-denominator three-isotope correlation diagrams (CDTIC), in use for 60 years. A complementary tool to identify mineral mixtures is the differential release of ${ }^{39} \mathrm{Ar},{ }^{38} \mathrm{Ar}$ and ${ }^{37} \mathrm{Ar}$, which reveals exactly the same mineralogical heterogeneities as EPMA and CDTIC. Reassessing literature studies supposed to illustrate ideal Fick's Law diffusion during in vacuo stepwise heating of ideally monomineralic micas documents that in actual fact a host of non-mica phases, degassed at very low and very high $\mathrm{T}$, bias and invalidate the inferred mathematical modelling of Ar release.

Anhydrous feldspars are not destabilized by dehydration. However, they are just as prone to retrograde reactions, such that EPMA, CL and TEM demonstrate that even "diffusion archetypes" consist of half a dozen different phases produced by chemically open-system reactions. Moreover, high-temperature Raman spectroscopy on the monomineralic museum-grade Madagascar sanidine reproduces the well-known observations by crystallographers that feldspars undergo both displacive and irreversible structural transformations upon heating. Indeed, the stepwise release during a $951 \mathrm{ks}$ isothermal degassing follows a sigmoidal curve instead of a straight line in a Fechtig-Kalbitzer diagram. Since the diffusion matrix is not inert, downslope extrapolation of high-T degassing rates is not representative of Ar loss at low $\mathrm{T}$ in natural environments, as the high- $\mathrm{T}$ structure is not identical to the low-T structure. 